\title{
Virtual wet lab practice: From a sudden crisis towards the deployment of an integrated faculty strategy
}

\author{
F. Selmin*, I. Eberini, P. Minghetti \\ School of Pharmacy, University of Milan/Università degli Studi di Milano, Italy
}

\author{
Keywords \\ Educational Experiences \\ Pharmaceutics \\ Student perceptions \\ Virtual Lab Practice \\ Italy
}

${ }^{*}$ Corresponding author: francesca.selmin@unimi.it
Summary: This is a brief overview on student engagement and perception of remote practical activities during the COVID-19 outbreak emergency. The topics that were heavily affected by these sudden and unexpected changes, where chemistry, biochemistry, bioinformatics, pharmacology, and compounding. This survey took responses from the students of both bachelor and master's degrees at the School of Pharmacy of the University of Milan. This University is in the epicentre of the COVID-19 outbreak in Italy. Despite a good or high appreciation of the online practical activities, up to $95 \%$ of participants agreed that multimedia content cannot efficiently replace inperson labs. Moreover, discussions with the teachers and discussions among lab mates has a great positive impact on the knowledge and skills they acquire.

\section{Background and Context}

The University of Milan 'La Statale' is located in Milan, the regional capital of Lombardy, where the largest Italian outbreak of COVID-19 was identified.

The teaching and learning paradigm implemented at the Faculty of Pharmacy is based mainly on traditional teaching methods, institutional lectures in classrooms and a practical section based on training laboratories. On-line teaching at the University has always been intended as an optional support activity to the traditional approach. This was the scenario, in March 2020, when the in-person lessons and activities were the banned in response to the COVID-19 outbreak. This persuaded the Italian government to lockdown Milan and, after a few days, to ban all 'nonessential movement' throughout the country.

In a matter of days, students and teachers had to completely reorganise their activities, update their lessons and methods to remote teaching and learning technologies. If classical lessons required a significant effort of customisation to try to overcome on-line critical issues, laboratory courses appeared to be the greatest challenge for teaching during this period of crisis. The aim of practical labs (both dry and wet labs) is not only to develop practical skills, but also represents a unique opportunity to forge a critical approach to the content taught in lectures and to create a network of interpersonal ties.

Some issues, moreover, have also arisen from the need to apply remote teaching methods to typically manual activities.

\section{Educational Description}

The measures taken by the Steering Committee of the School of Pharmacy were aimed at mitigating the delay in students' learning with the necessity to provide adequate learning content and support. The topics that were heavily affected by these sudden and unexpected changes, where chemistry, biochemistry, bioinformatics, pharmacology, and compounding. The two stage response for most of the lab courses for master's degrees has been as follows: the first step consists of a subsidy of about $66 \%$ of European Credit Transfer and Accumulation System (ECTS) using online education platforms to replace them where possible; the second step is in September 2020, the remaining ECTS would be completed by doing in-person practicals in labs which are currently under reorganisation to follow the COVID-19 regulations.

The online resources made available by several organisations and institutions have been useful but not enough to 
efficiently cover the teaching activities of the School of Pharmacy's highly-specialised classes, even with the useful multimedia content available on YouTube and other networks/sources (e.g. JoVE). As a positive and collaborative example, a set of videos on pharmacy compounding have been recorded by members of ADRITELF, the Italian organisation of the pharmaceutical technology and legislation of academics. Their main aim was to provide the key foundational principals of basic operations of pharmaceutical technology.

On the other hand, lab work in biochemistry courses is usually based on discussions about the proper application of analytical procedures, with the aim of better understanding laboratory medicine cases. To adapt this to COVID-19 emergency requirements, the University decided to use synchronous lessons to 'practically' apply all the base concepts studied during asynchronous lessons to real cases. In-depth discussions were carried out on each clinical case to encourage the development of students' critical approaches to cases.

At the end of the term, an anonymous non-institutional survey was sent out to students to evaluate their engagement and perceptions of the practical activities adapted as a result of the COVID-19 emergency. Indeed, student perceptions have been an important tool for assessing and improving the educational experience. In addition, allowing students to be included in the quality improvement efforts enabled their voices to be heard, which further enhanced their engagement within their educational environment (Bowman, 2020).

The survey included questions that asked students to indicate how beneficial each remote lab was to their learning experience using a four-point scale; how different their experience was with respect to in-person labs; and openended questions that asked students to provide comments. Completion of the questionnaire took about two to three minutes. The collected data was analysed in aggregate using Microsoft Excel for Microsoft 365 MSO.

The respondents were 425 students enrolled on both the bachelors and master's programmes. Generally speaking, the students expressed a good or high appreciation for the proposed activities, with higher scores for dry labs. About $60 \%$ of participants indicated a high appreciation (i.e. level three or higher in the four-point scale) and only $10 \%$ provided a negative evaluation. As expected, up to $95 \%$ of participants agreed that multimedia content cannot efficiently replace wet labs, especially the face-to-face experience shared with lab mates, instructors and teachers during practicals. In very few cases, this mindset became particularly evident when pre-assignments were attributed to students because they were considered 'extra-work' which prevented them from studying. However, the high appreciation, which was independent of the considered class and type of activity, was in line with the word frequency analysis carried out by building a tag cloud using all the words repeated more than once in the students' comments: 'distance', 'hand-on' and 'practice'. However, the frequent recurrence of 'achieve', 'surely', 'despite, 'situation' also pointed out that students positively recognised the efforts to propose alternative solutions in such a short and critical time, to reach the learning goals without a delay and experience an empathetic/sympathetic understanding of the efforts made by all the people involved in the teaching/learning system in the Faculty and more in general at the University.

\section{Outcomes and Recommendations}

Coping with the emotional stress of pandemic has shown that some boundaries between teachers and students need to be re-defined and new approaches and tools are required to start the upcoming academic year with many COVID-19 restrictions still in effect.

The response of the University of Milan 'La Statale' to the lockdown has demonstrated how technology can help to transform the way institutions teach and enable learning. Some of the creative solutions and tools acquired from this period may be integrated in a reshaped teaching strategy to make lessons and practice activities more efficacious. Moreover, the lag time between dry labs and in-person activities should also provide the opportunity for students to learn at their own pace and review the appropriate lecture material ahead of time in order to fully engage with the lab exercises. For structural bioinformatics labs, staff are exploring the possibility of making all of the computational software available for installation on students' computers, to allow them to test/reproduce all the proposed exercises in a completely virtual setup.

The current experience also underlines the tight connection between the student's participation and perception of educational experiences. Not only do pharmacy students consider all in-person activities essential requirements for their professional qualifications, but also that discussion with teachers and among lab mates is greatly importance in acquiring knowledge and skills.

\section{References}

Bowman, B.J. (2020). Improving students' perceptions of pre-lab assignments and corresponding group discussions within a pharmacy compounding lab. Pharmacy Education, 20(1), 179 - 190. https://doi.org/10.46542/pe.2020.201.179190 